\title{
A Pseudo Potential Description of the Motion of Charged Particles in RF Fields
}

\section{A.S. Berdnikov}

Institute for Analytical Instrumentation RAS, Rizskiy pr. 26, 190103 St.Petersburg, Russian Federation

\section{Introduction}

The pseudo potential model used to describe the 'averaged' motion of charged particles in radio frequency (RF) electric field is considered here [1-4]. This approach describes the motion in RF fields just qualitatively and cannot describe correctly the subtle effects like the true stability zone, etc. The pseudo potential model is very useful for intuitively transparent prediction of the motion of charged particles in the electric RF fields.

Classical pseudo potential approach deals mainly with stationary sinusoidal fields but here the superposition of sinusoidal fields with arbitrary frequencies and slowly evolving amplitudes is considered. The expressions for corresponding pseudo potential function are derived and analyzed. It appears that such RF fields allow new interesting ways to control the motion of the charged particles. Additional effects are disclosed when the motion of charged particles in a non-uniform and nonstationary gas-filled media is considered. However, to see some subtle details of the motion one should keep higher order terms in the equations - this converts the pseudo potential description of the motion into the pseudo Hamiltonian one. A brief description of these new possibilities mainly discovered by the author is represented in this paper.

\section{Background of the pseudo potential method}

The equations of motion of charged particle in radio frequency electric (rf) field is written as

$$
m \frac{d^{2} x_{k}}{d t^{2}}=-q\left(\frac{\partial U(\vec{x})}{\partial x_{k}}+\frac{\partial V(\vec{x})}{\partial x_{k}} \cos \omega t\right)
$$

where $m$ is the mass of the particle, $q$ is the charge of the particle, $\vec{x}=\left(x_{1}, x_{2}, x_{3}\right)$ are the geometrical coordinates, $U(\vec{x})$ is the static part of the electric potential, $V(\vec{x})$ is the amplitude of the rf part of the electric potential, $\omega$ is the frequency of the sinusoidal signal (i.e., voltages applied to the electrodes), $t$ is the time. The integration of (1) is a complex task and mainly can only be done numerically. However, it is difficult to make a transparent understanding what happens with a charged particle in the rf electric field using Eq. (1) as the main instrument because here the fundamental aspects of motion are masked by mathematical complexity.

A typical way how to introduce the pseudo potential model of charged particle motion is the following. First, the solution of Eq. (1) is represented as $\vec{x}(t) \approx \vec{y}(t)+\delta \vec{z}(t)$ where $\vec{y}(t)$ is the "slow" component of the solution and $\delta \vec{z}(t)$ is the fast component of the solution which is considered to be small correction to the main part $\vec{y}(t)$. Namely, it is assumed that

$\delta z(t) \approx \vec{z}_{c 1}(t) \cos \omega t+\vec{z}_{s 1}(t) \sin \omega t+\vec{z}_{c 2}(t) \cos 2 \omega t+\vec{z}_{s 2}(t) \sin 2 \omega t+\cdots$

where the amplitudes $\vec{z}_{c 1}(t), \vec{z}_{s 1}(t), \vec{z}_{c 2}(t), \vec{z}_{s 2}(t), \ldots$ are considered to be small as compared with the main part $\vec{y}(t)$ of the solution and it is assumed that both the function $\vec{y}(t)$ and the functions 
$\vec{z}_{c 1}(t), \vec{z}_{s 1}(t), \vec{z}_{c 2}(t), \vec{z}_{s 2}(t), \ldots$ are changing with a much larger characteristic time scale than the period $T=2 \pi / \omega$ of rf oscillations. Next step is to represent the amplitudes $\vec{z}_{c 1}(t), \vec{z}_{s 1}(t), \vec{z}_{c 2}(t), \vec{z}_{s 2}(t), \ldots$ as the Taylor series with respect to $1 / \omega$ (i.e., in the vicinity of the point $\omega=\infty$ ):

$$
\begin{aligned}
& \vec{z}_{c 1}(t) \approx \frac{1}{\omega} \vec{z}_{c 1}^{(1)}(t)+\frac{1}{\omega^{2}} \vec{z}_{c 1}^{(2)}(t)+\frac{1}{\omega^{3}} \vec{z}_{c 1}^{(3)}(t)+\cdots \\
& \vec{z}_{s 1}(t) \approx \frac{1}{\omega} \vec{z}_{s 1}^{(1)}(t)+\frac{1}{\omega^{2}} \vec{z}_{s 1}^{(2)}(t)+\frac{1}{\omega^{3}} \vec{z}_{s 1}^{(3)}(t)+\cdots .
\end{aligned}
$$

This can be done, since it is clear that near $\omega=\infty$ the rf component of the solution vanishes: due to the inertia the particle remains nearly in the same position providing the rf component of the electric field periodically changes its direction quickly enough. Then we can represent the potentials $U(\vec{y}+\delta \vec{z})$, $V(\vec{y}+\delta \vec{z})$ and their derivatives as Taylor series near the point $\vec{y}(t)$ since it is assumed that $\|\delta \vec{z}(t) \mid<<\| \vec{y}(t) \|:$

$$
\begin{aligned}
& \frac{\partial U(\vec{y}+\delta \vec{z})}{\partial x_{k}} \approx \frac{\partial U(\vec{y})}{\partial x_{k}}+\sum_{i} \frac{\partial^{2} U(\vec{y})}{\partial x_{k} \partial x_{i}} \delta z_{i}+\frac{1}{2} \sum_{i, j} \frac{\partial^{3} U(\vec{y})}{\partial x_{k} \partial x_{i} \partial x_{j}} \delta z_{i} \delta z_{j}+\cdots \\
& \frac{\partial V(\vec{y}+\delta \vec{z})}{\partial x_{k}} \approx \frac{\partial V(\vec{y})}{\partial x_{k}}+\sum_{i} \frac{\partial^{2} V(\vec{y})}{\partial x_{k} \partial x_{i}} \delta z_{i}+\frac{1}{2} \sum_{i, j} \frac{\partial^{3} V(\vec{y})}{\partial x_{k} \partial x_{i} \partial x_{j}} \delta z_{i} \delta z_{j}+\cdots
\end{aligned}
$$

Now we can combine Eqs. (1) to (4) and collect separately different harmonics $\cos k \omega t$, sin $k \omega t$ and different powers $1 / \omega^{n}$; this enables us to produce an explicit expressions for the unknown functions $\vec{z}_{c, k}^{(n)}(t), \vec{z}_{s, k}^{(n)}(t)$. Finally, the following compact equations for the "averaged" solution $\vec{y}(t)$ can be derived (where we keep the terms $1 / \omega^{2}$ and neglect terms of higher order):

$$
\frac{d^{2} y_{k}}{d t^{2}}=-\frac{q}{m} \frac{\partial U}{\partial y_{k}}-\frac{q^{2}}{2 m^{2} \omega^{2}} \sum_{j} \frac{\partial V}{\partial y_{j}} \frac{\partial^{2} V}{y_{j} \partial y_{k}}-\cdots
$$

The equations (5) do not look simpler than Eq. (1), however, they can be rewritten as the equations of motion of the particle with mass $m$ and charge $q$ in some artificial electric field:

$$
\frac{d^{2} y_{k}}{d t^{2}}=-\frac{q}{m} \frac{\partial}{\partial y_{k}}[U(\vec{y})+\bar{U}(\vec{y})] \text {, or } \frac{d^{2} \vec{y}}{d t^{2}}=-\frac{q}{m} \vec{\nabla}[U(\vec{y})+\bar{U}(\vec{y})]
$$

where the pseudo potential function $\bar{U}(\vec{y})$ is calculated as

$$
\bar{U}(\vec{y})=\frac{q}{4 m \omega^{2}} \sum_{j}\left(\frac{\partial V}{\partial y_{j}}\right)^{2}
$$

The investigation of the profile for the pseudo potential function $U(\vec{y})+\bar{U}(\vec{y})$ allows making conclusions about the motion of the charged particle without solving the equations (5): if there are some potential wells we can expect stable oscillations inside these wells; if there are the slopes going to infinity the motion is unstable. (While the original potential $U(\vec{x})+V(\vec{x}) \cos \omega t$ cannot have potential wells far from the electrodes due to the Laplace properties of the potential functions $U(\vec{x})$ and $V(\vec{x})$, the pseudo potential $U(\vec{y})+\bar{U}(\vec{y})$ can have such potential minima.) The redirecting of attention from the 
equations of motion (1) to the profiles of the pseudo potential functions (7) is the main point of the pseudo potential technique.

The method of the effective potential was first proposed by Kapitsa in the 1950s to calculate the stable modes of oscillations for a vertical pendulum with a vibrating suspension [5,6]; later he used this method to successfully solve some complex problems of high power electronics [7]. The concept of this method began with the fundamental works of Mitropolski and Bogoliubov summarized in [8]. Landau generalized as a formal method the original effective potential approach proposed by Kapitsa to describe generally mechanical motion of a particle in a rapidly time-oscillating potential force of an arbitrary type [1]. For the description of the motion of charged particles in systems with radio frequency electromagnetic fields the effective potential method was introduced as a general tool by Gaponov and Miller [9,10]. Many subsequent publications re-derived this in different ways; its specific features were analyzed in more detail and more carefully than in the original publications. Good reviews of these methods, in English, are presented in [3,11].

\section{Simple applications and further extensions of the pseudo potential method}

Simple examples of the usage of the pseudo potential technique are the stack ring ion guides [12-15]. Here the electrodes are a sequence of rings or planes with circular holes [15]. To the electrodes rf voltages $\pm U_{R} \cos (\omega t+\varphi)$ are applied where the neighboring electrodes always have potentials with opposite polarities:

$$
U_{2 n}(t)=+U_{R} \cos (\omega t+\varphi), \quad U_{2 n+1}(t)=-U_{R} \cos (\omega t+\varphi)
$$

The rf potential for the electric field inside the ion guide is defined as [16]:

$$
U_{R F}(z, r, t)=U_{R} \cdot\left[I_{0}\left(\frac{\pi r}{L}\right) / I_{0}\left(\frac{\pi R}{L}\right)\right] \cos \left(\frac{\pi z}{L}\right) \cos (\omega t+\varphi)
$$

where $r=\sqrt{x^{2}+y^{2}}$ is the radial coordinate, $z$ is the axial coordinate, $L$ is the distance between the electrodes, $R$ is the radius of the electrodes, $I_{0}$ is the modified Bessel function [17]. The profile of the pseudo potential calculated using Eq. (7) looks like a tray with rapidly (exponentionally) growing edges where it approaches the boundaries of the electrodes - the region with strongly inhomogeneous rf field. As a result the particles are well confined inside the ion guide. Additionally there is the sinusoidal corrugation along the tray which complicates the usage of the device as an effective mobility separator or mobility spectrometer [18]. A more sophisticated example of such a device is called "RF Carpet" which uses the same technique as described in ref. [19,20].

When the sinusoidal voltages applied to the electrodes have the same frequency but different phase values the rf electric field has a more complex representation:

$$
U_{r f}(\vec{x}, t)=U(\vec{x})+V(\vec{x}) \cos \omega t+W(\vec{x}) \sin \omega t
$$

or, equivalently,

$$
U_{r f}(\vec{x}, t)=U(\vec{x})+G_{R F}(\vec{x}) \cos \left(\omega t-\varphi_{R F}(\vec{x})\right)
$$

where $G_{R F}(\vec{x})=\sqrt{(V(\vec{x}))^{2}+(W(\vec{x}))^{2}}, \varphi_{R F}(\vec{x})=\arctan (W(\vec{x}) / V(\vec{x}))$. In this case the "slow" part of the trajectory is also defined by the equations (6) but with a different recipe how to calculate the pseudo potential component $\bar{U}(\vec{y})$ : 


$$
\bar{U}(\vec{y})=\frac{q}{4 m \omega^{2}} \sum_{j}\left(\left(\frac{\partial V}{\partial y_{j}}\right)^{2}+\left(\frac{\partial W}{\partial y_{j}}\right)^{2}\right)=\frac{q}{4 m \omega^{2}} \sum_{j}\left(\left(\frac{\partial G_{R F}}{\partial y_{j}}\right)^{2}+\left(G_{R F} \frac{\partial \varphi_{R F}}{\partial y_{j}}\right)^{2}\right)
$$

This extended expression can be used to eliminate the parasitic corruption of the pseudo potential profile for stack ring ion guides. Namely, when the voltages are applied as

$$
U_{4 n}(t)=+U_{R} \cos \omega t, \quad U_{4 n+1}(t)=+U_{R} \sin \omega t, \quad U_{4 n+2}(t)=-U_{R} \cos \omega t, \quad U_{4 n+3}(t)=-U_{R} \sin \omega t,
$$

the rf potential for the electric field inside the ion guide is defined as [21]

$$
U_{R F}(z, r, t)=U_{R} \cdot\left[I_{0}\left(\frac{\pi r}{2 L}\right) / I_{0}\left(\frac{\pi R}{2 L}\right)\right]\left(\cos \left(\frac{\pi z}{2 L}\right) \cos \omega t+\sin \left(\frac{\pi z}{2 L}\right) \sin \omega t\right)
$$

and the pseudo potential of Eq. (12) demonstrates no corrugation along the axis $O Z$. (Similar technique can be used for devices like rf carpets, ion funnels, etc., to eliminate or to decrease parasitic corrugations.)

The most general rf voltages which produce electric fields for which the pseudo potential technique can be used are the voltages described by the pseudo discrete Fourier spectrum [22]. In this case the spectrum is decomposed into islands with nonzero values while the spectrum outside the islands is exactly zero (Figure 1). The islands should be "narrow" and should be placed "far from each other" where the terms "narrow" and "far" are treated in the same way as "slow motion" and "fast frequency" for the classical pseudo potential theory.

(a)

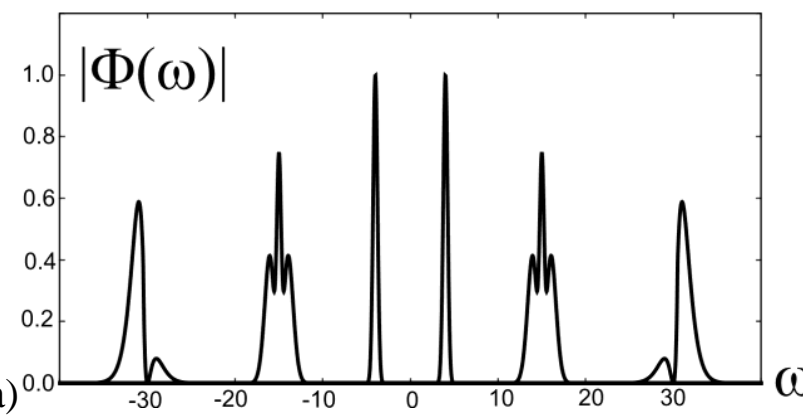

(b)

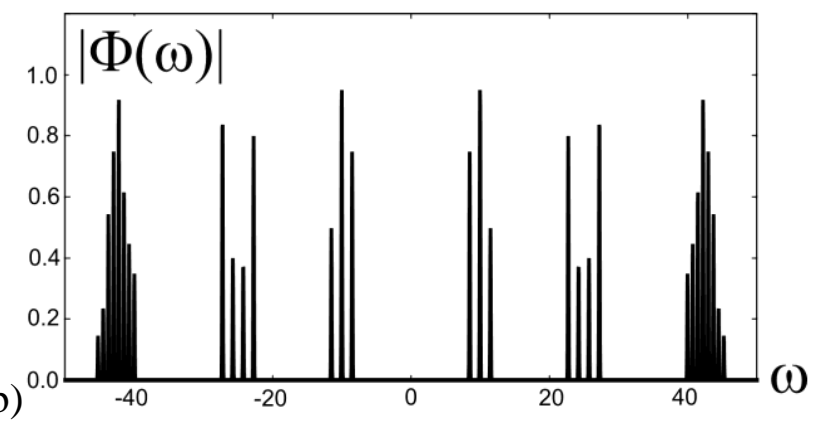

Figure 1. Examples of the pseudo discrete Fourier spectra: (a) continuous pseudo discrete spectrum, (b) pulsed pseudo discrete spectrum.

The voltages $f(t)$ which are characterized by the pseudo discrete spectrum can always be represented as the combination of fast sinusoidal signals with slowly evolving amplitudes:

$$
f(t)=\sum\left(p_{k}(t) \cos \omega_{k} t+q_{k}(t) \sin \omega_{k} t\right)
$$

which results to the rf electric potential represented as

$$
U_{r f}(\vec{x}, t)=\sum\left(V_{k}(\vec{x}, t) \cos \omega_{k} t+W_{k}(\vec{x}, t) \sin \omega_{k} t\right)
$$

The pseudo potential technique applied to Eq. (16) results in the equations (6) with the pseudo potential function which can be calculated as

$$
\bar{U}(\vec{y}, t)=\sum_{k} \frac{q}{4 m \omega_{k}^{2}} \sum_{j}\left(\left(\frac{\partial V_{k}(\vec{y}, t)}{\partial y_{j}}\right)^{2}+\left(\frac{\partial W_{k}(\vec{y}, t)}{\partial y_{j}}\right)^{2}\right)=\sum_{k} \frac{q}{4 m \omega_{k}^{2}}\left(\left|\vec{\nabla} V_{k}(\vec{y}, t)\right|^{2}+\left|\vec{\nabla} W_{k}(\vec{y}, t)\right|^{2}\right)
$$

The expression in Eq. (17) is close to that of Eq. (12), however, there is an essential difference here: the 
pseudo potential $\bar{U}(\vec{y}, t)$ depends on time and hence enables more possibilities to control the motion of charged particles. An example of the devices which use the pseudo potentials (17) are the A-Wave ion guides [23,24]. Here the charged particles with different charges and masses are collected by the rf electric field into compact bunches and these bunches of particles are moved from entry to exit with the same group velocity regardless of differences in masses, charges and initial energies for individual particles inside the bunch.

\section{Pseudo magnetic fields, pseudo Hamiltonians, etc.}

What we did up to now is to represent the solution $\vec{x}(t)$ of the equations of motion as $\vec{x}(t) \approx \vec{y}(t)+\delta \vec{z}(t)$ where $\vec{y}(t)$ is the "slow" component of the solution and $\delta \vec{z}(t)$ is the "fast" component of the solution and to find the equations for the "slow" function $\vec{y}(t)$ as a series with respect to $1 / \omega^{n}$ :

$$
\frac{d^{2} y_{k}}{d t^{2}}=(\cdots)+\frac{1}{\omega}(\cdots)+\frac{1}{\omega^{2}}(\cdots)+\frac{1}{\omega^{3}}(\cdots)+\frac{1}{\omega^{4}}(\cdots)+\cdots
$$

where the series (18) is restricted by the terms $1 / \omega^{2}$. However, it is interesting to see what happens if we restrict Eq. (18) to terms up to $1 / \omega^{3}, 1 / \omega^{4}$. etc.

The terms $1 / \omega^{3}$ are responsible for the appearance of the pseudo magnetic field $\vec{B}_{\text {eff }}(\vec{y}, t)$ if there is no other agent acting on the particle. However, if there is a true quasi static magnetic field $\vec{B}(\vec{y}, t)$ an additional pseudo potential term appears as well. The resulting equations for the averaged trajectory $\vec{y}(t)$, which can be derived using the same technique, are the following:

$$
\frac{d^{2} \vec{y}}{d t^{2}}=-\frac{q}{m} \vec{\nabla}\left[U(\vec{y}, t)+\bar{U}(\vec{y}, t)+\vec{B}(\vec{y}, t) \cdot \vec{H}_{e f f}(\vec{y}, t)\right]+\frac{q}{m}\left(\frac{d \vec{y}}{d t} \times\left[\vec{B}(\vec{y}, t)+\vec{B}_{e f f}(\vec{y}, t)\right]\right)
$$

where the pseudo potential function $\bar{U}(\vec{y}, t)$ is calculated as in Eq. (17) and

$$
\begin{aligned}
& \vec{H}_{\text {eff }}(\vec{y}, t)=\sum_{k} \frac{q^{2}}{2 m^{2} \omega_{k}^{3}}\left(\vec{\nabla} V_{k}(\vec{y}, t) \times \vec{\nabla} W_{k}(\vec{y}, t)\right), \quad \vec{B}_{\text {eff }}(\vec{y}, t)=\vec{\nabla} \times \vec{A}^{e f f}(\vec{y}, t), \\
& A_{i}^{\text {eff }}(\vec{y}, t)=\sum_{k} \frac{q}{2 m \omega_{k}^{3}}\left[\left(\vec{\nabla} \frac{\partial V_{k}(\vec{y}, t)}{\partial y_{i}}\right) \cdot\left(\vec{\nabla} W_{k}(\vec{y}, t)\right)-\left(\vec{\nabla} V_{k}(\vec{y}, t)\right) \cdot\left(\vec{\nabla} \frac{\partial W_{k}(\vec{y}, t)}{\partial y_{i}}\right)\right] .
\end{aligned}
$$

The terms $1 / \omega^{4}$ and higher result in very complex equations with no transparent interpretation. To simplify them it is necessary to convert the equations into the hamiltonian representation using some pseudo Hamiltonian function $\bar{H}=\bar{H}_{0}+\frac{1}{\omega} \bar{H}_{1}+\frac{1}{\omega^{2}} \bar{H}_{2}+\cdots$. Even more interesting is the consideration of motion of charged particles in a gas media taking into account the nonlinear character of the drag force coefficient, the existence of gas flows, spatial gradients of gas flows and gas media characteristics, rf phase spatial gradients. Accurate calculations result in the appearance of additional non-potential pseudo forces which are absent in the simplified damped pseudo potential model [25]. However, the detailed considerations of these interesting topics are outside the scope and the size of this paper and will be published separately. 


\section{Conclusion}

Pseudo potentials are an interesting challenge and wonderful toy for CPO theoreticians. Pseudo potentials are very useful in inventing new and powerful rf devices for mass spectrometry as well. The investigation of the pseudo potentials is definitely not finished and there are still many interesting facts in this field of science to be discovered by the inquisitive researches [26].

References:

[1] LD Landau and EM Lifshitz in "Mechanics", (Pergamon Press, Oxford, 1960) p. 93.

[2] RZ Sagdeev, DA Usikov and GM Zaslavsky in "Nonlinear Physics: From the Pendulum to Turbulence and Chaos", (Harwood Academic Publishers, New York, 1988).

[3] D Gerlich, in "State-Selected and State-to-State Ion-Molecule reaction Dynamics. Part 1:

Experiment”, eds. M Baer et al, (Wiley, New York, 1992) p. 1.

[4] MI Yavor in "Optics of Charged Particle Analyzers", (Elsevier, Amsterdam, 2009) p. 142.

[5] PL Kapitsa, ZhETF 21 (1951), p. 88.

[6] P L Kapitsa, Physics-Uspekhi 44 (1951), p. 7.

[7] PL Kapitsa, Physics-Uspekhi 78 (1962), p. 181.

[8] NN Bogoliubov and YA Mitropolski in "Asymptotic Methods in the Theory of Nonlinear Oscillations", (Hindustan, Delhi, 1961).

[9] VA Gaponov and MA Miller, ZhETF 34 (1958), p. 242.

[10] MA Miller, Izvestiya VUZov (Radiophysics) 1 (1958), p. 110 (in Russian).

[11] RF Wuerker, H Shelton and RV Langmuir, J. Appl. Phys. 30 (1959), p. 342.

[12] R Bahr, Diploma Thesis (University of Freiburg, 1969).

[13] D Gerlich, Diploma Thesis (University of Freiburg, 1971).

[14] E Teloy and D Gerlich, Chem. Phys. 4 (1974), p. 417.

[15] D Gerlich and G Kaefer, Astrophys. J. 347 (1989), p. 849.

[16] AV Tolmachev et al, Int. J. Mass Spectrom. 203 (2000), p. 31.

[17] GN Watson in "Treatise on the Theory of Bessel Functions", (Cambridge University Press,

Cambridge, 1966).

[18] K Giles et al, Rapid Comm. Mass Spectrom. 18 (2004), p. 2401.

[19] H Wollnik and Y Ueno, US Patent no. 8013296 (2011).

[20] A Takamine et al, Rev. Sci. Instrum. 76 (2005), 103503.

[21] AS Berdnikov and NR Gall, J. Anal. Chem. 69 (2014), p. 1285.

[22] AS Berdnikov, Doctoral Thesis (Institute for Analytical Instrumentation, St. Petersburg, 2013).

[23] AD Andreyeva and AS Berdnikov, J. Anal. Chem. 67 (2012), p. 1034.

[24] AS Berdnikov, AD Andreyeva and R Giles, Patent Application WO 2012/150351 (2012).

[25] AV Tolmachev et al, Nucl. Instr. Meth. B 124 (1997), p. 112.

[26] The author acknowledge Dr. Sumio Kumashiro and Dr. Mikhail Sudakov from Shimadzu Corp. for their inspirations and maintaining of his interest to rf systems and Prof. Hermann Wollnik for his many important and useful consulting. 